\title{
Constraints on micro-physical parameters of GRBs using HAWC
}

\author{
Simone Dichiara ${ }^{* a}$, Maria Magdalena González ${ }^{a}$, Nissim Fraija $^{a}$ for the HAWC \\ collaboration ${ }^{e}$ \\ ${ }^{a}$ Instituto de Astronomía, Universidad Nacional Autónoma de México, México D.F., México \\ ${ }^{e}$ For a complete author list, see http://www.hawc-observatory.org/collaboration/icrc2017.php.
}

E-mail: soichiara@astro.unam.mx

The physical mechanism at the origin of gamma-ray bursts (GRBs) is far from being completely understood. Describing their emission up to very high energies $(\mathrm{GeV}-\mathrm{TeV})$ is one of the most challenging and important tasks needed to unveil the physics of these peculiar events. Using data collected by the HAWC gamma-ray observatory, we search for $\mathrm{TeV}$ emission coming from a sample of GRBs detected by Fermi and Swift between December 2014 and May 2017. We derive upper limit over different time intervals and use them to constrain the micro-physical parameters and the bulk Lorentz factor under the assumption of Self-Synchrotron Compton within the external shock model scenario. We present the results of this analysis discussing possible interpretations.

7th Fermi Symposium 2017

15-20 October 2017

Garmisch-Partenkirchen, Germany

\footnotetext{
*Speaker.
} 


\section{Introduction}

The Large Area Telescope instrument on board the Fermi satellite (Fermi/LAT, [1]) revealed a high-energy component (>100 MeV) from over $100 \mathrm{GRBs}^{1}$. This emission lasts more and shows a different spectral evolution [2]) than the one observed at lower energies during the prompt phase. The study of the high energy emission coming from GRBs could reveal important information about emission mechanisms and physics of these peculiar phenomena. Moreover, it allows to probe the effects of the extra-galactic background light (EBL; see [3]), to derive the initial Lorentz factor of the jet and to estimate the Lorentz invariance violation (see e.g. [4]). High energy photons could be originated when the jet interacts with the external medium accelerating electrons which scatter synchrotron radiation (synchrotron self-Compton; hereafter SSC) by the shock wave up to energies from $\mathrm{GeV}$ to $\mathrm{TeV}$. In general, the external medium can present a constant density profile (e.g. interstellar medium; ISM, see[5]), a stellar wind profile (e,g [6, 7, 8]) or a combination of these two (e.g. [9]). According to Meszaros and Rees [10], the inverse Compton scattering could be the dominant cooling mechanism of accelerated electrons in the external shock wave when it gets close to the deceleration radius. In this work we compare the theoretical predictions for the SSC emission at high energy with the flux upper limits derived for a sample of GRBs within the field of view of the High Altitude Water Cherenkov observatory (HAWC). We use the HAWC data to test the external shock model, probe the circumburst medium and constrain the micro-physical parameters involved in this scenario.

\section{HAWC}

HAWC is an extensive air shower array located at Sierra Negra, in the state of Puebla, Mexico. This observatory uses a water Cherenkov technique to study $\mathrm{TeV} \gamma$-ray radiation and cosmic rays. The experiment has a field of view of $\sim 2$ str and it is continuously operating (24/7 monitoring) since March 2015. It covers $\sim 2 / 3$ of the sky every sidereal day being particularly suitable for GRB follow up at very high energies. The events or air showers detected by HAWC are classified in ten different size bins (from 0 to 9 ) which are based on the fraction of available PMT activated by the event as described by [11]. The lowest bin (bin 0) is excluded from this analysis.

\section{Data Analysis}

We search for $\mathrm{TeV}$ emission at the position of 108 GRBs within the field of view of HAWC from December 2014 to May 2017. Twenty-one of them are short GRBs $\left(T_{90}{ }^{2}<2\right.$ seconds) and eighty-seven are long ones ( $T_{90} \geq 2$ seconds). Eighty out of 108 GRBs were observed by the Gamma-Ray Burst Monitor on board of Fermi (GBM). Four of them were co-detected at higher energies by Fermi/LAT. For 28 GRBs, more precise information on the position was provided by the Swift satellite. We estimate the significance of the signal in different time window durations. $T_{90}$ is used for long GRBs and a fix duration of 2 seconds for the short ones. In order to explore

\footnotetext{
${ }^{1}$ https://fermi.gsfc.nasa.gov/ssc/observations/types/grbs/lat_grbs/

${ }^{2} T_{90}$ is defined as the time during which the cumulative number of detected counts increases from $5 \%$ to $95 \%$ above background, thus encompassing $90 \%$ of the total GRB counts
} 
possible late time signal we also estimate the significances in ten consecutive time windows of the same duration for each GRB. Flux upper limits in the energy range $80-800 \mathrm{GeV}$ are derived for each time window following the same procedure outlined in [12] using Monte Carlo simulations (see [11]) to obtain the HAWC effective area. We assume a power law spectrum considering two different spectral indices predicted for fast, index of -1.5 , and slow cooling, index of -1.7 , regimes. Then, we introduce the attenuation effects due to the EBL according to the fiducial model proposed by [13].

We focus our analysis on short GRBs since HAWC is more sensitive to this specific subclass (see [14]). The bulk of sGRB are between redshift of 0.1 and 1.3, with an average value of 0.5 (see [15]). Therefore, we assume a conservative value of 1 for the redshift when we account for the EBL effects.

\subsection{Model}

The dynamics of the external shock for the ejecta expanding into a surrounding medium with homogeneous density has been widely explored [9]. Using the synchrotron spectra, the evolution of synchrotron energy breaks and the maximum flux, the light curve in the fast-cooling regime is proportional to $\propto t^{-\frac{3 p-2}{4}} E^{-\frac{p}{2}}$ for $E_{\gamma, \mathrm{m}}^{\mathrm{syn}}<E^{\mathrm{syn}}$ and $\propto t^{-\frac{1}{4}} E^{-\frac{1}{2}}$ for $E_{\gamma, \mathrm{c}}^{\mathrm{syn}}<E^{\mathrm{syn}}<E_{\gamma, \mathrm{m}}^{\mathrm{syn}}$, where $E_{\gamma, \mathrm{c}}^{\mathrm{syn}}$ and $E_{\gamma, \mathrm{m}}^{\mathrm{syn}}$ are the synchrotron spectral breaks for the cooling and characteristic energies, respectively [5]. Relativistic electrons accelerated in the forward shocks could scatter synchrotron photons up to energies larger than $100 \mathrm{GeV}$. The SSC spectral breaks (the characteristic $\left(E_{\gamma, \mathrm{m}}^{\mathrm{ssc}}\right)$, the cooling $\left(E_{\gamma, \mathrm{c}}^{\mathrm{ssc}}\right)$ and the Klein-Nishina $\left(E_{\gamma, \mathrm{c}}^{\mathrm{KN}}\right)$ energies) as well as the maximum SSC flux are in the form

$$
\begin{aligned}
& E_{\gamma, \mathrm{m}}^{\mathrm{ssc}} \propto \varepsilon_{e}^{4} \varepsilon_{B}^{1 / 2} n^{-1 / 4} E^{3 / 4} t^{-9 / 4} \quad E_{\gamma, \mathrm{c}}^{\mathrm{ssc}} \propto \varepsilon_{B}^{-7 / 2} n^{-9 / 4} E^{-5 / 4} t^{-1 / 4}, \\
& E_{\gamma, \mathrm{c}}^{\mathrm{KN}} \propto \varepsilon_{B}^{-1} n^{-3 / 4} E^{-1 / 4} t^{-1 / 4} \quad F_{\gamma, \max }^{\mathrm{ssc}} \propto \varepsilon_{B}^{1 / 2} n^{1 / 4} D^{-2} E^{5 / 4} t^{1 / 4} .
\end{aligned}
$$

where $n$ is the homogenous density, $D$ is the luminosity distance, $E$ is the isotropic equivalent kinetic energy, $\varepsilon_{e}$ and $\varepsilon_{B}$ are the micro-physical parameters defined as fraction of the energy density that goes to accelerate electrons and to amplify the magnetic field, respectively. Taking into consideration $E_{\gamma, \mathrm{c}}^{\mathrm{ssc}} \leq E_{\gamma} \leq E_{\gamma, \mathrm{m}}^{\mathrm{ssc}}$, the flux as a function of time in the corresponding energy regime is given by

$$
F_{v}^{\mathrm{ssc}} \propto \varepsilon_{B}^{-5 / 4} n^{1 / 8} D^{-2} E^{5 / 8} t^{1 / 8} E_{\gamma}^{-1 / 2}
$$

\section{Results and Discussion}

The distribution of the significances extracted in each time windows for all the GRBs is displayed in Fig. 1. As observed, no statistically significant detection is found. Therefore upper limits for the flux are derived.

The most constraining upper limits are the corresponding to GRB 170206A. For this case, the fluence upper limits are comparable or below the fluence measured by GBM during the prompt emission in the energy range of 10-1000 keV. GRB 170206A is the third brightest short burst detected by Fermi-GBM. Flux upper limits derived for this burst at different times for $\mathrm{z}=1$ are shown in Fig. 2.

These limits can be used to constrain the normalization of equation 3.2, thus the allowed range for the micro-physical parameters $\varepsilon_{B}$ and $\varepsilon_{e}$ assuming different values for $\mathrm{n}$ (and $\Gamma$ ). Fig. 3 shows 


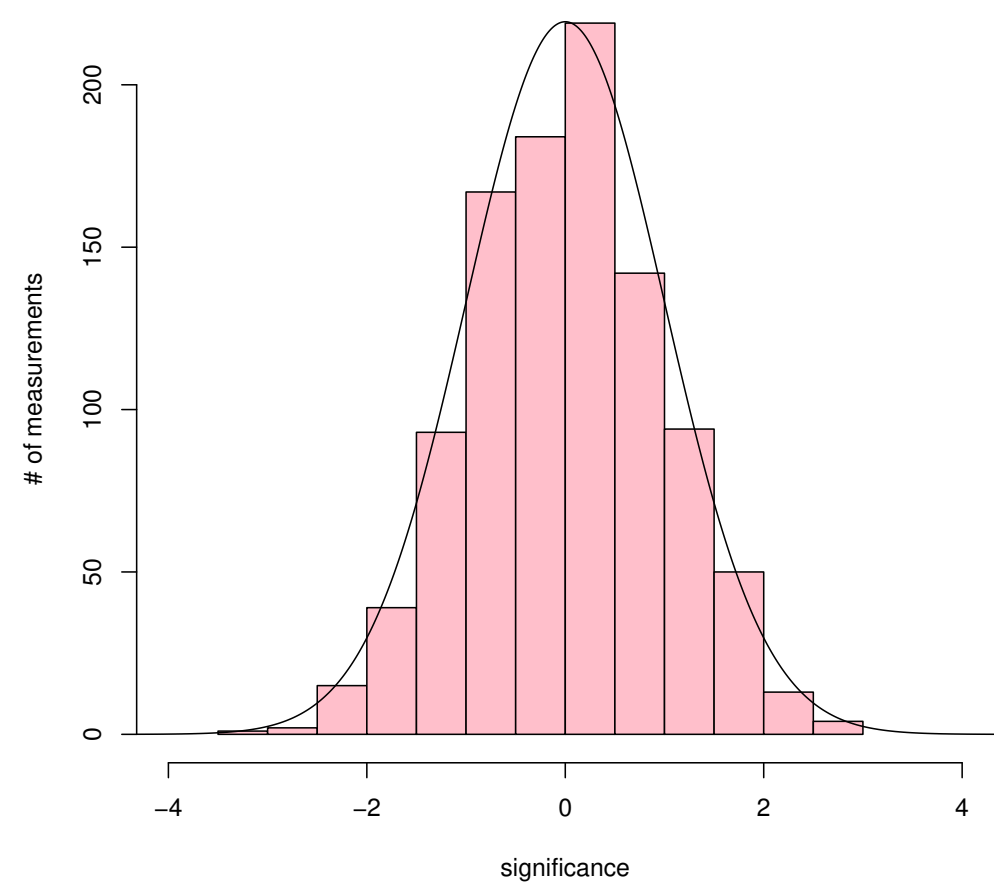

Figure 1: The plot shows the distribution of the significances derived over 10 time windows for each GRB of our sample. It is consistent with a standard normal distribution. The solid line shows a Gaussian function centered at 0 and standard deviation of 1 .

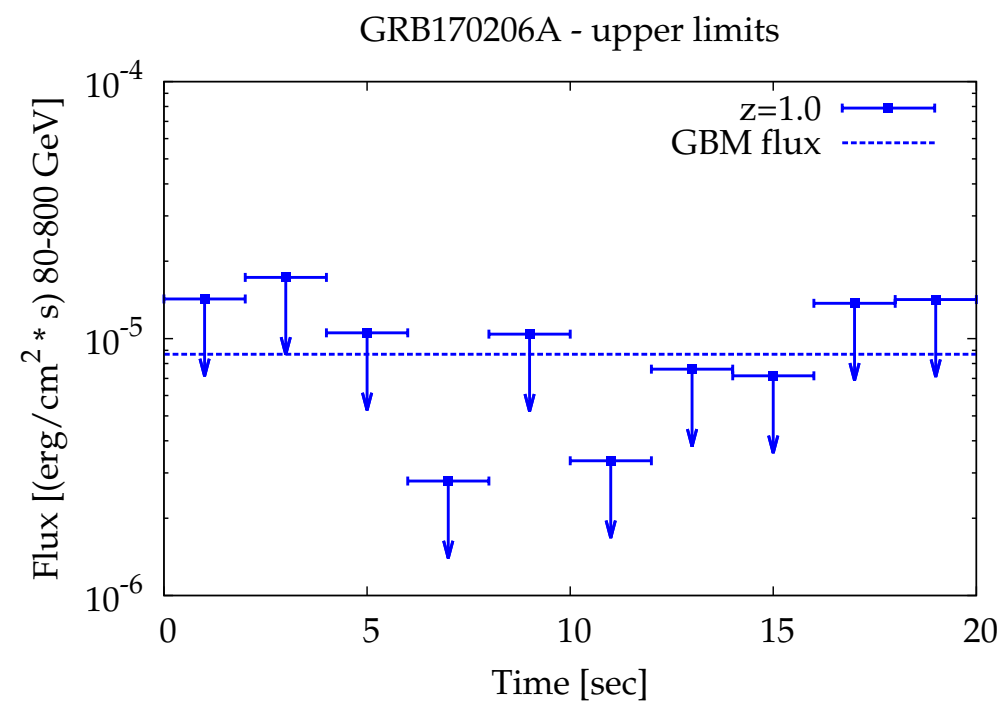

Figure 2: Flux upper limits extracted for GRB 170206A over different time windows in the energy range 80-800 GeV assuming a redshift of 1 (blue arrows). The average flux measured by Fermi-GBM in the energy range $10-1000 \mathrm{keV}$ is shown for comparison (dashed blue line). 
the allowed values for $\varepsilon_{B}$ and $\varepsilon_{e}$ derived assuming $\mathrm{n}=0.5 \mathrm{~cm}^{-1}$ and considering the upper limits measured for GRB $170206 \mathrm{~A}$ at $\mathrm{z}=1$ in the fast cooling regime. We find an upper limit for ISM density of $\mathrm{n}<1.1 \mathrm{~cm}^{3}$ in order to the model in the fast cooling regime be consistent with the limits measured by HAWC. On the other hand, assuming the slow cooling regime, we find that the density have to be lower than 12 particles per $\mathrm{cm}^{3}$.

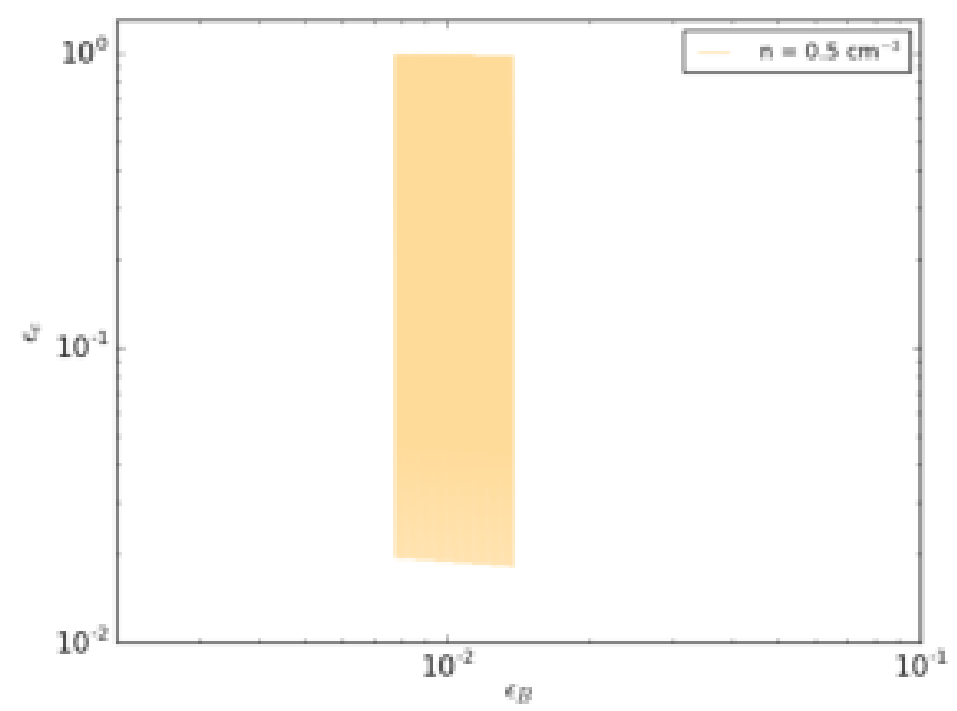

Figure 3: The allowed range for the micro-physical parameters $\varepsilon_{B}$ and $\varepsilon_{e}$ is derived using the GRB 170602A flux upper limits and assuming a fast cooling regime at redshift 1.0. The allowed region would be smaller assuming lower values of $\mathrm{z}$ (detailed results will be shown in a future work currently in progress). A density of 0.5 particles per $\mathrm{cm}^{3}$ is considered $(\Gamma=1150)$

\section{Conclusions}

Using the HAWC data we search for TeV emission from 108 GRBs occurred between December 2014 and May 2017. No evidence of GRB TeV emission is found neither at early or late times. Upper limits for the flux and fluence are derived for each GRB. We use the limits derived for GRB 170206A to constrain the micro-physical parameters and to probe the prediction proposed for the VHE emission in the framework of the external shock model. Assuming a fast cooling regime, the density of the ISM is found to be $\mathrm{n}<1.1 \mathrm{~cm}^{-3}$ which imply a limit on the relativistic Lorentz factor of $\Gamma=\left(\frac{3}{32 \pi m_{p}}\right)^{1 / 8}(1+z)^{3 / 8} n^{-1 / 8} E^{1 / 8} t^{-3 / 8}>1000$. For the slow cooling regime, a lower limit for the density of $\mathrm{n}<12 \mathrm{~cm}^{-3}$ is found $(\Gamma>800)$. The detection of new constraining upper limits together with a multi-wavelength analysis would provide an important tool to test the physics of the GRB afterglow and to probe the nature of the VHE emission.

\section{Acknowledgments}

We acknowledge the support from: the US National Science Foundation (NSF); the US Department of Energy Of- 
fice of High-Energy Physics; the Laboratory Directed the US National Science Foundation (NSF); the US Department of Energy Office of High-Energy Physics; the Laboratory Directed Consejo Nacional de Ciencia y Tecnología (CONACyT), México (grants 271051, 232656, 260378, 179588, 239762, 254964, 271737, 258865, 243290, 132197 281653), Laboratorio Nacional HAWC de rayos gamma; L'OREAL Fellowship for Women in Science 2014; Red HAWC, México; DGAPA-UNAM (grants IG100317, IN111315, IN111716-3, IA102715, 109916, IA102917); VIEP-BUAP; PIFI 2012, 2013, PROFOCIE 2014, 2015; the University of Wisconsin Alumni Research Foundation; the Institute of Geophysics, Planetary Physics, and Signatures at Los Alamos National Laboratory; Polish Science Centre grant DEC2014/13/B/ST9/945; Coordinación de la Investigación Científica de la Universidad Michoacana. Thanks to Scott Delay, Luciano Díaz and Eduardo Murrieta for technical support.

\section{References}

[1] B. W. Atwood, A. A. Abdo, M. Ackermann, et al. The Large Area Telescope on the Fermi Gamma-Ray Space Telescope Mission, ApJ 697 (2009) 1071-1102

[2] M. Ackermann, M. Ajello, K. Asano, et al. The First Fermi-LAT Gamma-Ray Burst Catalog, ApJS 209 (2013) 11

[3] M. Schirber and J. S. Bullock Faint Active Galactic Nuclei and the Ionizing Background, ApJ 584 (2003) 110-128

[4] G. Amelino-Camelia , J. Ellis, N. E. Mavromatos, et al Tests of quantum gravity from observations of $\gamma$-ray bursts, Nature 393 (1998) 763-765

[5] N. Fraija , W. H. Lee, P. Veres and R. Barniol Duran Modeling the Early Afterglow in the Short and Hard GRB 090510, ApJ 831 (2016) 22

[6] N. Fraija GRB 110731A: Early Afterglow in Stellar Wind Powered By a Magnetized Outflow, ApJ 804 (2015) 105

[7] N. Fraija , W. H. Lee, P. Veres Modeling the Early Multiwavelength Emission in GRB130427A, ApJ 818 (2016) 190

[8] N. Fraija , W. H. Lee, M. Araya, P. Veres, R. Barnio-Duran and S. Guiriec Modeling the high-energy emission in GRB 110721A and implications on the early multiwavelength and polarimetric observations, arXiv 1709.06263 (2017)

[9] N. Fraija et al. Theoretical Description Of GRB 160625B with Wind-to-ISM Transition and Implications for a Magnetized Outflow, ApJ 848 (2017) 15

[10] P. Meszaros and M. Rees Delayed GEV Emission from Cosmological Gamma-Ray Bursts - Impact of a Relativistic Wind on External Matter, MNRAS 269 (1994) L41

[11] A. U. Abeysekara, A. Albert, R. Alfaro, et al. Observation of the Crab Nebula with the HAWC Gamma-Ray Observatory, ApJ 843 (2017) 39

[12] R. Alfaro, C. Alvarez, J. D. Álvarez, et al. Search for Very-high-energy Emission from Gamma-Ray Bursts Using the First 18 Months of Data from the HAWC Gamma-Ray Observatory, ApJ 843 (2017) 88

[13] R. C. Gilmore, R. S. Somerville, J. R. Primack, et al. Semi-analytic modelling of the extragalactic background light and consequences for extragalactic gamma-ray spectra, MNRAS 422 (2012) 3189-3207

[14] I. Taboada and R. C. Gilmore Prospects for the detection of GRBs with HAWC, NIMPA 742 (2014) 276

[15] E. Berger Short-Duration Gamma-Ray Bursts, ARA\&A 52 (2014) 43-105 